\title{
IMAGING AND CONTROL FOR ADAPTIVE RADIOTHERAPY
}

\author{
Olivier Haas*, Gloria Bueno+, Ralf Spriestersbach*, Heiko Himmler*, Keith Burnham*, John Mills ${ }^{\mathrm{x}}$ \\ *Control Theory and Applications Centre, Coventry University CV1 5FB, UK \\ + University Castilla-La Mancha, E.T.S.I Industriales, Avda. Camilo José Cela - 13071 Ciudad Real, E. \\ ${ }^{\mathrm{x}}$ University Hospitals Coventry and Warwickshire NHS Trust, Coventry, UK,
}

\begin{abstract}
This paper reviews existing techniques to deliver radiotherapy treatment and describes the requirements from a control engineering view point to adapt radiation delivery to organs motion. The first part describes recent evolution of radiotherapy research; the second part focuses on imaging modalities used in radiotherapy; the third part reviews image processing techniques to detect organ movement; the final section compares traditional PID with predictive control strategies for use in patient positioning devices. Copyright (C) 2005 IFAC
\end{abstract}

Keywords: medical applications, control system design, image processing, robot, PID control.

\section{INTRODUCTION}

This paper aims to present the challenges and opportunities, from a control engineering perspective, arising from new radiotherapy treatment techniques. Until recently radiotherapy treatment could have been considered, from a control engineering view point, as an open loop process with some form of feed-forward. Indeed, traditionally radiotherapy treatment was delivered based on treatment plans calculated from images of the patient's internal anatomy taken before the treatment. This means that it was not possible or practical to predict at any time during the actual treatment delivery the particular position of the patient's internal body structures, nor the dose actually received by these tissues. Instead, some form of open loop feed-forward control was used, where clinicians based their treatment schedules on predicted effect of radiation on the cancerous cells and on 'expected' location of the organs. This necessitated the addition of margins to the cancerous structures. The clinical target volume accounts for uncertainty and microscopic extent of the tumour. To reduce such margin imaging modalities able to measure the activity of cancerous tissues are used, see (Mackie, et al., 2003) for a good review of imaging modalities for radiotherapy applications. The planning target volume (PTV) accounts for organ movements (Mackie, et al., 2003). Such margins increase the dose delivered to healthy tissues, resulting in increased risk of treatment complications and limiting the dose that can be delivered to the cancerous regions. From the end of the 1980 s to the late 1990s the focus of research in radiotherapy was on the optimisation of the modulation of X-ray beams to deliver a dose of radiation that conforms to the tumours and spares healthy tissues. These techniques are referred to as conformal radiotherapy and intensity modulated radiation therapy (IMRT) (Haas, 2003). Such advances resulted in improved treatment and demonstrated the ability to reduce the dose to healthy tissues dramatically, whilst at the same time allowing an increased dose delivery to the PTV. The development of new IMRT treatment delivery machines means that today clinicians are able to 
deliver the appropriate dose of radiation, to treat cancer much more accurately than ever before. One of the remaining issues preventing IMRT to reach its full potential is directly linked with the actual detection of the cancer location before and during treatment. Significant work has been carried out in medical imaging to detect cancerous structures and to distinguish normal body structures, see for example (Bueno et. al, 2004, 2002). However, until recently, it was not possible to use any 'feedback' with respect to the actual position of the target and its evolution. Indeed, to account for organ movements it is necessary to detect these in real time.

The advent of megavoltage imaging, such as electronic portal imaging devices (EPID), created significant enthusiasm. EPID enables clinicians and physicists to locate 'anatomical landmarks' whilst the radiation beam used to treat the cancer is active, (Stroom, et al., 2000). EPID is, however, only able to distinguish regions which are significantly different from a density view point. For example bones can be identified, but it is very difficult to differentiate soft tissues. In addition, when an intensity modulated beam is delivered the image produced by EPID device is 'corrupted' by the non uniform amount of radiation that strikes the detector. The latest developments in medical imaging, allow the reconstruction of good quality 3D images of the patient anatomy within the radiotherapy treatment suite (Jaffray, et al., 2002). This means that some form of dynamic feedback control is now possible. Indeed, the position of the patient and/or the position of the radiotherapy apparatus can be adjusted from the information provided by the imaging devices present in the radiotherapy treatment suite. Such types of treatments are referred to as adaptive radiation therapy (ART), 4-dimensional IMRT, where the fourth dimension is time (Mackie, et al., 2003) or image guided radiation therapy.

Section 2 describes the facilities required for 4-D IMRT. Section 3 reviews the techniques used to identify and track regions of interest within the patient. Section 4 focuses on the Elekta Synergy system which is taken as an example for the development of a new control system to position the patient following information taken prior treatment.

\section{IMAGING DEVICES FOR 4-D IMRT}

To deliver 4-D IMRT treatments it is necessary to eliminate variations between imaging, planning and treatment stage. The ultimate aim is to automatically locate the cancerous tissues and the healthy tissues and adapt the treatment to deliver a radiation solely to the cancerous tissues. To date a number of approaches have been developed. The simplest approaches use existing equipment such as, for example, a fluoroscopic camera based electronic portal imaging device (EPID) to verify the patient position prior treatment. Such system can be used to compare several 2-D images known as digitally reconstructed radiographs (DRR) generated from computed tomography (CT) scans with the images acquired by the EPID (Clippe, et al., 2003). EPID can also be used to assess the patient position whilst the treatment beams are delivered using algorithms such as that described in (Fielding, et al., 2002) to compensate for the non uniformity of the treatment beam and extract patient density information. Similarly, EPID can be used to monitor the dose delivered to the patient, hence providing some dose feedback (Kirby and Williams, 1995). To be useful, however the processing speed of the information collected by the EPID needs to be improved.

In (Meeks, et al., 2003) a standard ultrasound probe fitted with optical markers is tracked using an infrared vision system from (RadioCameras, ZMed Inc., Ashland, MA, USA). This system allows the reconstruction of 3-D ultrasound from the set of images obtained by a clinician manipulating the probe. Such a system allows to re-calculate the target displacement prior treatment.

The approaches, adopted by Varian Medical Systems Inc. and Elekta Ltd., the two major manufacturers of radiotherapy treatment suites, combine, on the same gantry the traditional linear accelerator with a kilovoltage X-ray imaging device. Such imaging devices (Varian On Board Imager ${ }^{\mathrm{TM}}$ and Elekta Synergy ${ }^{\circledR}$, see Figure 1) can be used for radiography, fluoroscopy and more importantly cone beam CT. The 3-D images produced by cone beam CT are generated by rotating the gantry around the patient and acquiring $\mathrm{X}$-ray images. These images are then reconstructed accounting for deflexion of the support arms for the X-ray source and the detector as well as gantry movements. The advantage of such a system is that is acquires 3-D images, that can be registered with the imaging used at the diagnostic and the planning stage, with the patient being positioned using the same patient support system (PSS) as that used during treatment. A similar system currently being developed at Harvard Medical School, USA and partially funded by Varian uses two sets of X-ray sources and fast amorphous silicon panels. Details of the design of the integrated radiotherapy imaging system (IRIS) can be found in (Berbeco, et al., 2004). To improve the patient clearance under the treatment machine, imaging devices can be fixed on the ceiling and/or the floor. Some imaging devices monitor external patient movements using 3D camera systems using two or more cameras whilst others use X-ray tubes to determine the location of internal body structures. Vision RT Ltd offers two 3D cameras in their work in progress product 'Alignrt' to match the patient surface to previously acquired scan. POLARIS optical tracker systems (Northern Digital Inc, Waterloo, Canada) have been used in a number of applications in radiotherapy to track patient 
movements using a combination of an infrared tracking system and reflective markers, see for example (Tomé, et al., 2001, Tang, et al., 2004). In (Spriestersbach, et al., 2004) the POLARIS system was used to provide independent measurement to establish the accuracy of the PSS being developed. The advantage of the POLARIS system is that it is portable and can therefore be moved to a position that prevents interference from other devices.

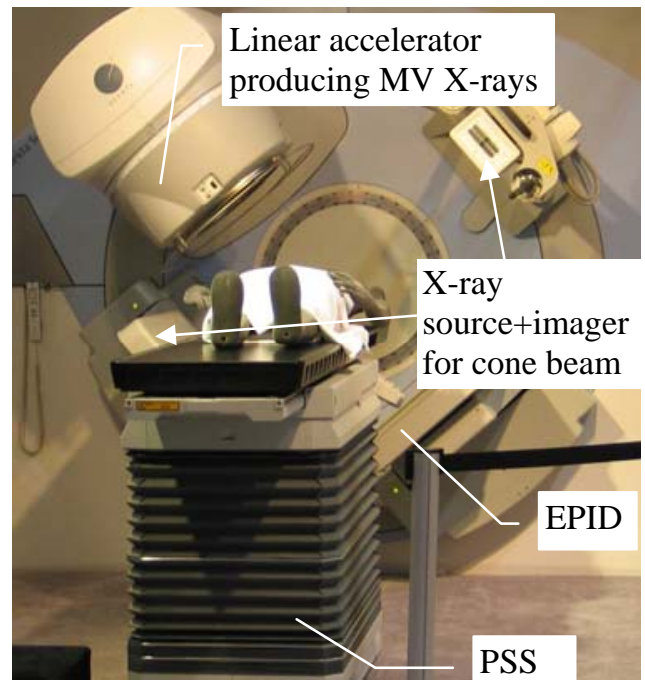

Fig. 1. Illustrating the use of a patient support system to position a patient during treatment with the Elekta Synergy® (Courtesy of Elekta Ltd).

A real time tumour tracking radiotherapy (RTRT) system has been developed to track gold markers (Kitamura, et al., 2002, Shirato, et al., 2004). It uses fluoroscopy image processor units to determine the $3 \mathrm{D}$ position of the implanted marker. The RTRT device comprises two concurrent sets of 2 imaging devices fixed to the ceiling and two X-ray sources fixed onto the floor. According to (Shirato, et al. 2004) such a set up does not interfere as much with the actual treatment delivery as the PSS can be moved freely under the radiation beam. The manufacturers previously mentioned may argue that the X-ray tube and flat panel imager (imaging system recording the X-ray transmission through the patient) could be retracted before treatment. The third approach includes, in the same room, a standard CT scanner and a linear accelerator sharing the same PSS onto which the patient is positioned. In (Onishi, et al., 2003) the CT scanner is moved using rails to image the patient. Once the CT scan has been acquired, the PSS is then rotated $180^{\circ}$ such that the part of the patient that was imaged can receive the required dose of radiation. In (Court, et al., 2003, Shiu, et al., 2003,) a similar set up is utilised with the exception that the PSS is rotated by $90^{\circ}$. In the latter it was found that the main source of uncertainty was the PSS position on the CT side after rotation. Finally, the last approach is based on the concept of tomotherapy. Tomotherapy Hi·Art System ${ }^{\circledR}$ aims to offers true 3D image guidance before each treatment
(Ruchala, et al., 2002). It is also envisaged in (Mackie, et al., 2003) that the CT scan during the treatment delivery will be used to reconstruct the dose delivered, hence providing another source of feedback. This brief review has highlighted the potential offered by new imaging systems that can be integrated with radiotherapy treatment devices. Video based tracking system are becoming readily available and have the advantage of being non invasive. X-ray bases system are subjecting the patient to additional radiation, however they have the advantage of being able to identify the internal body structured. These techniques should offer the capability to adapt the treatment depending both on the actual position of the organs to be treated and on the dose delivered to these organs and surrounding healthy tissues. Section 3 describes some of the image processing tools that are used to provide a set point to follow as well as a feedback signal.

\section{IMAGE PROCESSING FOR 4-D IMRT}

In 4-D IMRT image processing aims to automatically identify and then track regions of interest ROI, especially the PTV. Motion capture by anatomical structures segmentation and tracking are being used to address an increasingly number of medical applications. They are still a major challenge for image-guided therapy. Segmentation is an ill-posed problem, due to noise from the acquisition device, pose and reflection properties of the object together with their topology constraints. Some of these aspects may be considered known. Model-based segmentation methods encode such prior knowledge in the form of parametric representations. In the absence of prior knowledge, the use of model-free techniques based on geometric flows, statistical methods, Markov random fields, graph theory and region-growing tools have been investigated (Duncan et al., 2000, Bueno et al. 2002). Snake-driven approaches are popular in medical image segmentation. B-splines, deformable templates, Fourier descriptors are common ways to describe the structure of interest. Level set representations is an emerging technique to represent shapes and track moving interfaces for segmentation and tracking (Osher et al., 2003). The advantages of using a level set representation is that the algorithm can handle changes in the topology of the shape as the surface evolves in time and it can account for prior shape knowledge. Deformable models such as the thin-plate spline have also been used in (Schaly et a.l, 2004) to model organ deformation over the course of radiotherapy and highlight dose discrepancy between planned and delivered due to the organ motion.

Tracking is complementary to segmentation task that involves the recovery of the anatomical structure in the temporal domain. While such consideration can increase complexity, at the same time the use of dynamic information can improve segmentation 
performance. Tracking is equivalent to registration that is mapping pixel-wise correspondence, thus estimating the apparent motion. Optical flow estimation is required to establish correspondence from one frame to the next. Global motion models are a compromise between low complexity and good matching for planar objects (Murphy, 2004). To provide appropriate 'position' feedback for radiotherapy it is planned to investigate a level set framework for shape-driven knowledge-based, (Osher et al., 2003). In addition, a priori probabilistic information based on image statistical analysis, can be exploited. The display will be done through a boundary and region-based segmentation technique while internal constraints will be also imposed. Temporal information is accounted by seeking for a global motion model for the anatomical structure under consideration that satisfies the topological constraint.

\section{POSITION CONTROL FOR 4-D IMRT}

Having established the means to determine the control equivalent to a 'set point' and 'trajectory' to follow, this Section focuses on technical issues linked with set point following, i.e. tracking of organ motion and the adaptation necessary to take into account such motion in the treatment delivery. Organ motion can be dynamically compensated by i) moving the patient positioned onto a PSS, ii) adapting the leaves positions within a multileaf collimator, iii) moving the gantry, iv) tilting the PSS (Hornick et al., 1998). The remainder of this paper focuses issues related to (re)positioning the patient using the computer controlled PSS within Elekta Synergy ${ }^{\circledR}$ system. Until recently little control engineering research has been carried out to design modern control systems for radiotherapy applications. Most manufacturers rely on tried and tested approaches making use of proportional control or variants of proportional + integral + derivative (PID) control. The first part of this work was to design, develop and implement a standard control strategy with, however, some application specific features. The initial work presented in (Spriestersbach, et al., 2004), has now been refined and implemented in the PSS control within the new Elekta Synergy® system. This control scheme, which was financed through a Teaching Company Scheme between Coventry University and Elekta Ltd, will be used as a benchmark against which to assess the future control strategies. The authors believe that more advanced control techniques may be used to advantage to design automatic patient positioning that can adapt to different patient weight and resulting varying frictions or to move a gantry at an appropriate speed to allow effective computation of cone beam CT. Preliminary simulations have been carried out to assess the feasibility of model based control techniques. To design a model based control system, it is necessary to devise a representative model of the system. In (Spriestersbach, et al., 2003, Spriestersbach, et al., 2004) a SimMechanics model was developed using information from computer aided design drawings of the PSS and manufacturers datasheet. SimMechanics rigid bodies were linked together using joints, springs, dampers and gears. Finally, actuators and sensors were included to provide an interface with other Simulink blocks and toolboxes. The mechanical system is represented by a set of masses that simulate the inertia of the mechanical system components. This system recreates the characteristics of the motor-gearbox combination and is able to translate a given input voltage into the related force or torque that is applied to the moving parts of the system.

In this work, the model describing the PSS movement in the horizontal plane ( $\mathrm{X}$ and $\mathrm{Y}$ axes) and the vertical axis ( $\mathrm{Z}$ axis), (Spriestersbach, et al., 2003, Spriestersbach, et al., 2004), has been extended to include the rotation around the isocentre, see Figure 2. The I-module is similar to the $\mathrm{X}$ and $\mathrm{Y}$ modules with the SimMechanics ${ }^{\mathrm{TM}}$ prismatic joint replaced by a rotation joint, the torque to force conversion is replaced by a torque to torque conversion and the measurement in mm replaced by degrees. The means to model varying stiction or friction with respect to table-top speed and load have been added in the $\mathrm{X}$ and $\mathrm{Y}$ axes. Unlike in reality the prismatic and rotation joint blocks in SimMechanics are ideal, frictionless bearings between two bodies. As a consequence an applied force will be completely converted into acceleration. A more realistic friction/stiction has been implemented in the actuator such that the force/torque delivered by the motor/gearbox subsystem is reduced by the following stiction and friction forces:

- Kinetic friction force, subtracted to the external actuator force, the product of the reaction force caused by weight of table load and acceleration in the direction of the displacement with the kinetic friction coefficient.

- Forward and Reverse stiction limit, i.e. the force limits that must be exceeded for changing from the locked state (table stops) to the unlocked state (table moves) in forward and reverse direction. They are the product of the reaction force by the stiction coefficient.

- Static friction is the sum of the computed force (derived from the acceleration along the prismatic direction) and the actuator force: If it exceeds one of the stiction limits the table starts moving and the stiction disappears.

The model of the measurement subsystem has also been improved to include:

- quantization steps caused by the finite resolution of the digital-to-analog (DAC) converter,

- backlash caused by the DAC,

- measurement noise caused by electromagnetic interferences 
- Timing error 'noise' caused by small delays between a measurement and the time at which it is available for further processing.

The SimMechanics model is used in place of the actual PSS. Whilst being a good model from a simulation view point, it is too complex to be computed on line as part of a control strategy. Using this simulation platform, model structures applicable to control are derived. In this preliminary work, consideration was given solely to the $\mathrm{X}$ axis which is used to translate the table top and the patient. The Xaxis was approximated by a second order system which parameters are function of the aggregate mass of the table top and the patient. This second order model was then used to devise a i) lag compensator able to accommodate for varying patient mass and ii) an incremental generalized minimum variance (IGMV) control law. Figure 4 presents preliminary results, where the top left plot shows the control signal when a step of $10 \mathrm{~mm}$ is applied to the $\mathrm{x}$-drive unit, the top right plot shows the output. The bottom plot shows the trajectory followed by the $\mathrm{x}$-drive unit when the movement profile generator is used to modify the set point. It can be observed on the step responses that both the lag compensator and the IGMV controller can provide a more consistent performance with respect to changes in patient and table-top mass. The lag compensator has also the advantage of reducing the overshoot. In practice the PSS motion is restricted by the movement profile generator in terms of speed and acceleration to ensure optimal patient comfort. The bottom plot of Figure 4 shows that in all cases the controllers are able to achieve zero steady state error and no overshoot. Note that for confidentiality reasons the PID illustrated in this work is not representative of that implemented in the Elekta Synergy ${ }^{\circledR}$ system.

\section{CONCLUSIONS AND FURTHER WORK}

This paper has presented the potential of feedback control for radiotherapy treatments to compensate for organ/patient motion as well as dose actually delivered. Such control has been made possible by the recent developments in medical imaging, in terms of software, hardware as well as new dosimetry systems. The paper has reported on the implementation of a new PID based controller for the patient support system (PSS) within the Elekta Synergy ${ }^{\circledR}$ radiotherapy treatment suite. A SimMechanics model of the PSS has been refined to include the effect of nonlinearities. This model has subsequently been used to assess the potential of lag compensation and integral generalised minimum variance to compensate for varying pay load. Further work will aim to develop a new model based control scheme to compensate both for varying patient sizes and weight but also for varying frictions.

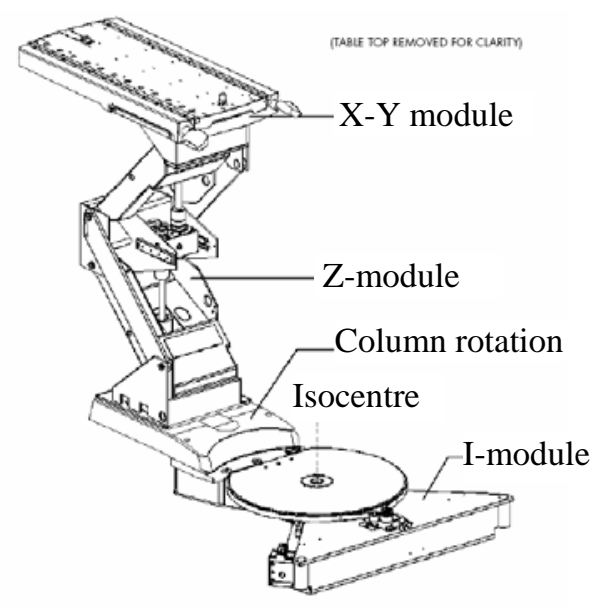

Fig. 2: Illustrating the PSS mechanical design (Courtesy of Elekta Ltd)

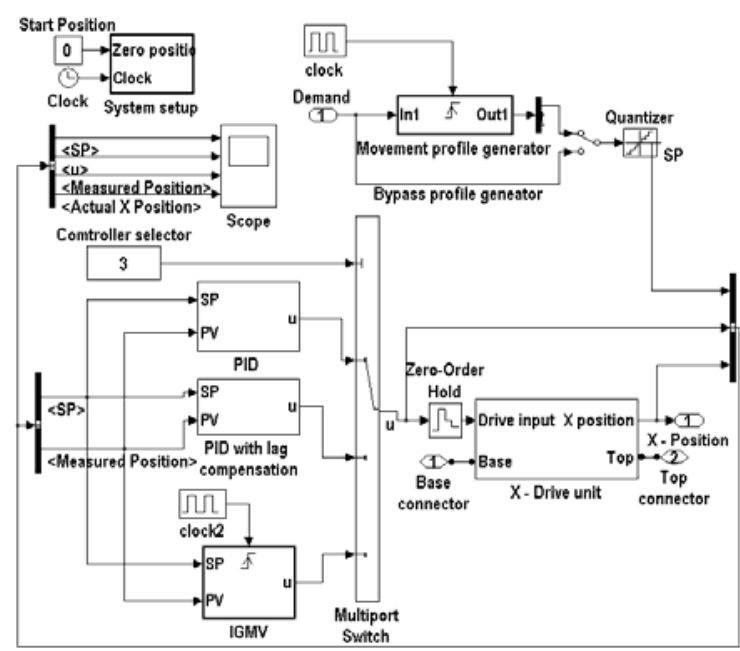

Fig. 3: Illustrating the evaluation of candidate control schemes for the X-axis SimMechanics model.
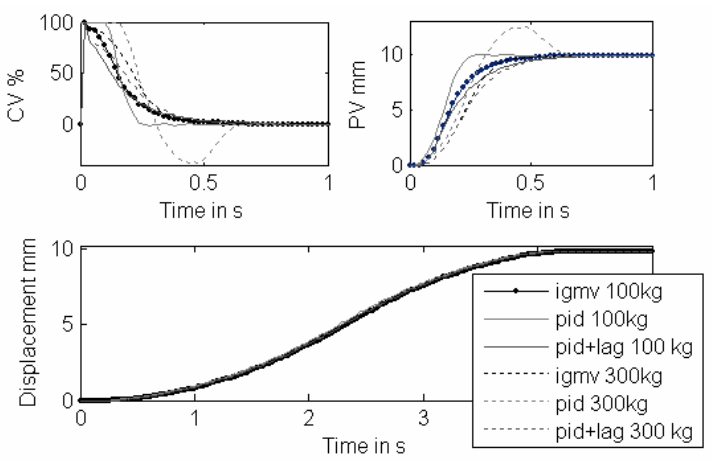

Fig. 4: Illustrating the benefit of model based control

\section{ACKNOWLEDGEMENTS}

The work presented in this paper is supported by the TCS Grant No 3787 and the Framework 6 European integrated project Methods and Advanced Equipment for Simulation and Treatment in Radiation Oncology (MAESTRO) CE LSHC CT 2004503564. 


\section{REFERENCES}

Berbeco, R.I., S.B. Jiang, G.C. Sharp, G.T. Chen, H. Mostafavi, H. Shirato (2004). Integrated radiotherapy imaging system (IRIS): design considerations of tumour tracking with linac gantry-mounted diagnostic x-ray systems with flat-panel detectors. Phys Med Biol, 49(2):24355.

Bueno G., Martínez-Albalá A., Adán A. (2004). Fuzzy-Snake Segmentation of Anatomical Structures Applied to CT Images, Lecture Notes in Computer Science; 3212(II), 33-42.

Bueno G., Heitz F., Armspach JP. (2002). 3D Segmentation of Anatomical Structures in MRIs on Large Data Bases, Journal of Magnetic Resonance Imaging, 19(1), 73-81.

Clippe S, Sarrut D, Malet C, Miguet S, Ginestet C, Carrie C. (2003). Patient setup error measurement using 3D intensity-based image registration techniques. Int $J$ Radiat Oncol Biol Phys, 56(1):259-65.

Court, L.E. and L.Dong (2003). Automatic registration of the prostate for computedtomography-guided radiotherapy, Medical Physics 30, (10), 2750-2757.

Duncan J.S., Ayache N. (2000). Medical Image Analysis: Progress over Two Decades and the Challenges Ahead. IEEE Trans. PAMI 22, 85-106.

Haas, O., 2003, An Intelligent Oncology Workstation for the 21st Century, Nowotwory Journal of oncology, Volume 53, Number 4, 389-397, PL ISSN 0029-540X

Hornick DC, Litzenberg DW, Kam KL, et al. A tilt and roll device for automated correction of rotational setup errors. Med Phys 1998;25:1739_ 1740.

Jaffray, D.A., J.H. Siewerdsen, J.W. Wong, A.A. Martinez (2002). Flat-panel cone-beam computed tomography for image-guided radiation therapy, Int J Radiat Oncol Biol Phys, 53(5), 1337-49.

Kirby, M.C., and P.C. Williams (1995). The use of an electronic portal imaging device for exit dosimetry and quality control measurements. Int $J$ Radiat Oncol Biol Phys; 31, 593-603.

Mackie, T.R.; J.Kapatoes; K. Ruchala; et al. (2003). Image guidance for precise conformal radiotherapy, Int J Radiat Oncol Biol Phys, 56(1), 89-105.

Meeks, S.L., J.M. Buatti, L.G. Bouchet, et al. (2003). Ultrasound-guided extracranial radiosurgery: technique and application, Int $J$ Radiat Oncol Biol Phys 55(4), 1092-101.

Murphy MJ. (2004) Tracking moving organs in real time, Semin. Radiat. Oncol. 14(1):91-100.

NDI Polaris, http://www.ndigital.com, (accessed 15/07/04).

Onishi, H., K. Kuriyama, T. Komiyama, et al. (2003). A new irradiation system for lung cancer combining linear accelerator, computed tomography, patient self-breath-holding, and patient-directed beam-control without respiratory monitoring devices. Int J Radiat Oncol Biol Phys ;56(1), 14-20.

Osher S. and Paragios N. (2003). Geometric Level Set Method in Imaging, Vision and Graphics, Springer.

Schaly, B., J.A. Kempe, G.S. Bauman, J.J.Battista, Van Dyk J. (2004). Tracking the dose distribution in radiation therapy by accounting for variable anatomy, Phys Med Biol, 49, 791805.

Shirato, H., M. Oita, K. Fujita, S. et al. (2004). Three-dimensional conformal setup (3D-CSU) of patients using the coordinate system provided by three internal fiducial markers and two orthogonal diagnostic X-ray systems in the treatment room. Int J Radiat Oncol Biol Phys. 60(2), 607-612

Shiu, A.S., E.L. Chang, J.S. Ye, et al. (2003). Near simultaneous computed tomography imageguided stereotactic spinal radiotherapy: an emerging paradigm for achieving true stereotaxy. Int $J$ Radiat Oncol Biol Phys. 57(3):605-13.

SimMechanics, 2004, The MathWorks (accessed 0302-2004) http://www.mathworks.com/

Smitsmans, M.H., J.W. Wolthaus, X. Artignan, J. et al. (2004). Automatic localization of the prostate for on-line or off-line image-guided radiotherapy. Int $J$ Radiat Oncol Biol Phys, 60(2), 623-35.

Spriestersbach R., O.C.L. Haas, K.J. Burnham, J. Allen (2003). Modelling of a patient positioning system: A precursor to control systems design, Proc 16th Int Conf. on Systems Eng., ICSE2003, Coventry, UK, 9-11 Sept 2003, 2:852-855.

Spriestersbach, R., O.C.L. Haas, K.J. Burnham, (2004) Modelling and control of patient support system for radiotherapy, Control 2004, Bath, UK, ISBN 086197130 2, 86 (abstract, CDROM).

Stroom JC, Olofsen-van Acht MJJ, Quint S, et al. (2000). On-line setup corrections during radiotherapy of patients with gynecologic tumors. Int J Radiat Oncol Biol Phys;46, 499-506.

Tai, P., J.Van Dyk, E. Yu, et al. (1998). Variability of target volume delineation in cervical esophageal cancer. Int J Radiat Oncol Biol Phys, 42, 227-288.

Tanga, J., S. Dieterichb, K. Clearya, (2004). Respiratory Motion Tracking of Skin and Liver in Swine for CyberKnife Motion Compensation, Medical Imaging 2004, May 2004, : Visualization, Image-Guided Procedures, and Display Robert L. Galloway, Jr. (Ed) 5367, CDS118, ISBN 0-81945295-5 (CDROM).

Tomé, W.A., S.L. Meeks, T.R. McNutt et al. (2001). Optically guided intensity modulated radiotherapy. Radiother Oncol, 61, 33-44. 